\title{
A Low-Cost Ambulance Idle Reduction System
}

\author{
Kevin S. Kung, PhD, EMT-B; Harrison J. Kaplan, EMT-B; Kyle J. Kotowick, EMT-B; \\ Charles S. Montgomery, SB, EMT-B
}

\section{POSTER PRESENTATION ABSTRACT}

Introduction: As per standard ambulance protocol, when the ambient temperature is below a certain threshold, it is necessary to keep the ambulance engine running while outside (i.e. "idling") because of the need to keep the cabin warm using the engine-heated heating system. This tends to be expensive in terms of fuel consumption and operating costs, and can also wear down the engine. While existing idle reduction technologies exist, they are often adapted from other applications such as fire apparatuses or police vehicles, which can have somewhat different functional requirements than ambulances. Such systems tend to be expensive and/or require intensive battery storage and monitoring. Program Development \& Implementation: By studying the non-emergent operations of a collegiate ambulance service (MIT EMS), we realized that in this particular case, most idling intervals are short (less than 30 minutes) relative to the timescale of heat loss from the patient compartment, and that the need for idling is mainly driven by temperature management rather than power management. This has enabled us to propose a simple, automated system design that constantly monitors the temperature in the patient compartment, and only starts the vehicle engine and heating system temporarily when the temperature falls below a preset threshold in the cabin. Program Evaluation: The simplest embodiment of this design, consisting of a remote starter operating in cold start mode and a modified temperature sensor, costs approximately one-sixth $(-\$ 750)$ compared to currently commercially available systems to install. A preliminary prototype system was installed on the MIT EMS ambulance (gas engine), and from the initial testing data, we estimate that our system can reduce the vehicle's idling fuel consumption and engine run time by about $85 \%$. Discussion/Conclusion: Future work includes studying the system's compatibility with diesel engine, adding dynamic control for engine run time and heater setting, and exploring the needs and requirements of other ambulance services.

Author Affiliations: Emergency Medical Services, Massachusetts Institute of Technology, Cambridge, MA, USA

Address for Correspondence: Kevin Kung, PhD, EMT-B E-mail: anti-idling@mit.edu

Conflicts of Interest/Funding Sources: By the JCEMS Submission Declaration Form, all authors are required to disclose all potential conflicts of interest and funding sources. The authors declared that they have no conflicts of interest. The authors declared that they received funding from Massachusetts Institute of Technology (MIT) Earth Day Sustainability Fund and MIT Emergency Medical Services for the program and/or research described in this abstract.

Ethical Compliance: The authors attest that the research associated with this abstract was conducted in accordance with the JCEMS Ethics Guidelines.

Submission History: Received January 18, 2018; accepted for presentation and publication February 22, 2018.

Poster Presentation: This abstract was presented as a poster at the Academ- ic Poster Session of the $25^{\text {th }}$ Annual Conference of the National Collegiate Emergency Medical Services Foundation; February 24, 2018; Philadelphia, PA, USA. The poster is available for download on the JCEMS website.

Published Online: May 15, 2018

Published in Print: August 13, 2018 (Volume 1: Supplemental 1)

Reviewer Information: In accordance with JCEMS editorial policy, poster presentation abstracts undergo double-blind peer-review by at least two reviewers (JCEMS Editorial Board members and/or independent reviewers) prior to acceptance for presentation and publication. JCEMS thanks the anonymous reviewers who contributed to the review of this work.

Copyright: (c) 2018 Kung, Kaplan, Kotowick, \& Montgomery. This is an open access abstract distributed under the terms of the Creative Commons Attribution 4.0 International (CC BY 4.0) License, which permits unrestricted use, distribution, and reproduction in any medium, provided the original author and source are credited. The full license is available at: https://creativecommons.org/licenses/by/4.0/

Electronic Link: https://doi.org/10.30542/JCEMS.2018.01.S1.10 